Supporting Information for: JA0555163

\title{
Asymmetric Addition of Secondary Phosphines to Methacrylonitrile: Catalysis and Mechanism
}

Aaron D. Sadow and Antonio Togni

Department of Chemistry and Applied Biosciences, Swiss Federal Institute of Technology, ETH Hönggerberg, CH-8093 Zürich, Switzerland

togni@inorg.chem.ethz.ch

$\left[\mathbf{N i}\left(\kappa^{3}\right.\right.$-Pigiphos)(methacrylonitrile $\left.)\right]\left[\mathbf{B A r}_{\mathrm{f}}\right]_{2} \cdot\left[\mathrm{NiBr}\left(\kappa^{3}-\mathrm{Pigiphos}\right)\right]\left[\mathrm{BAr}_{\mathrm{f}}\right]$ and $\mathrm{AgBAr}_{\mathrm{f}}$ were dissolved in a mixture of THF and methacrylonitrile (1:1). After $10 \mathrm{~min}$, a white precipitate formed. The solution was filtered and the solvents were removed in vacuo. Dissolution of the resulting powder gave a purple solution of $\left[\mathrm{Ni}\left(\mathrm{K}^{3}-\right.\right.$ Pigiphos)(methacrylonitrile)][BAr $]_{2}$. The dicationic portion was characterized in solution by ${ }^{1} \mathrm{H}$ and ${ }^{31} \mathrm{P}\left\{{ }^{1} \mathrm{H}\right\}$ NMR as identical to the dicationic portion of the crystallographically characterized $\left[\mathrm{Ni}\left(\kappa^{3}\right.\right.$-Pigiphos)(methacrylonitrile) $]\left[\mathrm{ClO}_{4}\right]_{2} .{ }^{1} \mathrm{H}$ NMR $\left(\mathrm{THF}-d_{8}, 330 \mathrm{~K}, 400\right.$

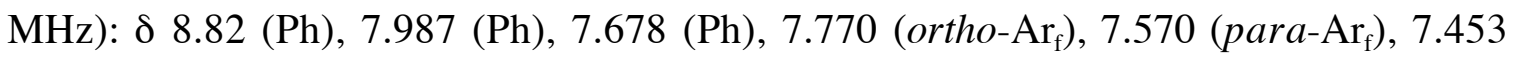
$(\mathrm{Ph}), 5.457\left(2 \mathrm{H}, \underline{\mathrm{H}}_{2} \mathrm{CCMeCN}\right) .5 .208\left(\mathrm{~s}, \mathrm{C}_{5} \mathrm{H}_{3}\right), 4.998\left(\mathrm{~s}, \mathrm{C}_{5} \mathrm{H}_{3}\right), 4.888\left(\mathrm{~s}, \mathrm{C}_{5} \mathrm{H}_{3}\right), 4.856(\mathrm{~s}$, $\left.\mathrm{C}_{5} \mathrm{H}_{3}\right), 4.791\left(\mathrm{~s}, \mathrm{C}_{5} \mathrm{H}_{3}\right), 4.511\left(\mathrm{~s}, \mathrm{C}_{3} \mathrm{H}_{5}\right), 4.167\left(\mathrm{~s}, 5 \mathrm{H}, \mathrm{C}_{5} \mathrm{H}_{5}\right), 3.930\left(\mathrm{~s}, 5 \mathrm{H}, \mathrm{C}_{5} \mathrm{H}_{5}\right), 3.740$ (m, $\left.1 \mathrm{H}, \mathrm{P}(\mathrm{C} \underline{\mathrm{HMeFc}})_{2}\right), 3.471\left(\mathrm{~m}, 1 \mathrm{H}, \mathrm{P}(\mathrm{C} \underline{\mathrm{HMeFc}})_{2}\right), 2.394(1 \mathrm{H}, \mathrm{Cy}), 2.207$ (dd, $3 \mathrm{H}$, $\left.\mathrm{P}(\mathrm{CHMeFc})_{2}, \mathrm{~J}=7.2,3.4 \mathrm{~Hz}\right), 1.997\left(\mathrm{dd}, 3 \mathrm{H}, \mathrm{P}(\mathrm{CHMeFc})_{2}, \mathrm{~J}=15.5,7.1 \mathrm{~Hz}\right), 1.541(2$ $\mathrm{H}, \mathrm{Cy}), 1.330(4 \mathrm{H}, \mathrm{Cy}), 1.030(2 \mathrm{H}, \mathrm{Cy}), 0.888(2 \mathrm{H}, \mathrm{Cy}), 0.581(1 \mathrm{H}, \mathrm{Cy}) .{ }^{13} \mathrm{C}\left\{{ }^{1} \mathrm{H}\right\}$ NMR (THF- $d_{8}, 330 \mathrm{~K}, 100 \mathrm{MHz}$ ): $\delta 162.27$ (q, ipso-Ar $\left.\mathrm{f}_{\mathrm{f}}, \mathrm{J}=49.9 \mathrm{~Hz}\right), 135.38(\mathrm{Ph})$, 135.08 (ortho- $\left.\mathrm{Ar}_{\mathrm{f}}\right), 134.26(\mathrm{Ph}), 133.99(\mathrm{Ph}), 133.57(\mathrm{Ph}), 133.32(\mathrm{Ph}), 132.05(\mathrm{Ph})$,

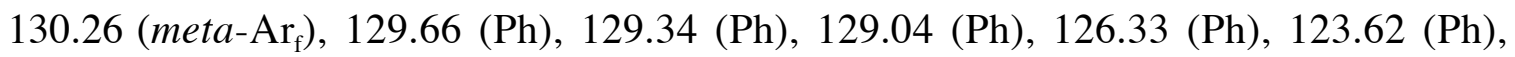
$120.92(\mathrm{Ph}), 117.57\left(\right.$ para $\left.-\mathrm{Ar}_{\mathrm{f}}\right), 89.56\left(\left(\mathrm{P}(\underline{\mathrm{CHMeFc}})_{2}\right), 89.40\left(\mathrm{P}(\underline{\mathrm{CHMeFc}})_{2}\right), 75.46\right.$ $\left(\mathrm{C}_{5} \mathrm{H}_{3}\right), 74.46\left(\mathrm{C}_{5} \mathrm{H}_{3}\right), 73.15\left(\mathrm{C}_{5} \mathrm{H}_{3}\right), 72.30\left(\mathrm{C}_{5} \mathrm{H}_{3}\right), 72.06\left(\mathrm{C}_{5} \mathrm{H}_{5}\right), 71.80\left(\mathrm{C}_{5} \mathrm{H}_{5}\right), 71.68$ $\left(\mathrm{C}_{5} \mathrm{H}_{3}\right), 71.59\left(\mathrm{C}_{5} \mathrm{H}_{3}\right), 71.55\left(\mathrm{C}_{5} \mathrm{H}_{3}\right), 71.50\left(\mathrm{C}_{5} \mathrm{H}_{3}\right), 71.40\left(\mathrm{C}_{5} \mathrm{H}_{3}\right), 71.31\left(\mathrm{C}_{5} \mathrm{H}_{3}\right), 54.03$ $\left(\mathrm{H}_{2} \underline{\mathrm{CCMeCN}}\right.$ ), $40.23(\mathrm{~d}, \alpha-\mathrm{Cy}, \mathrm{J}=17.5 \mathrm{~Hz}), 33.79$ (br m, Cy), 29.18 (d, Cy, J = 5.7 Hz), 
26.98 (d, Cy, J = 12.80 Hz), 25.95 (d, Cy, J = 9.4 Hz), $14.52\left(\left(\mathrm{P}(\mathrm{CHMeFc})_{2}, \mathrm{~J}=7.1 \mathrm{~Hz}\right)\right.$. ${ }^{31} \mathrm{P}\left\{{ }^{1} \mathrm{H}\right\}$ NMR (THF- $\left.d_{8}, 162 \mathrm{MHz}\right): \delta 87$ (br, CyP), 20 (br. $\left.\mathrm{PPh}_{2}\right), 12\left(\right.$ br, $\left.\mathrm{PPh}_{2}\right)$.

Figure S1. Representative pseudo-first-order plot $\left(\ln \left\{\left[t-\mathrm{Bu}_{2} \mathrm{PH}\right]_{0} /\left[t-\mathrm{Bu} \mathrm{u}_{2} \mathrm{PH}\right]_{t}\right\}\right.$ vs time $)$ of the $\left[\mathrm{Ni}\left(\kappa^{3} \text {-Pigiphos)(methacrylonitrile)][BAr }\right]_{2}\right.$-catalyzed reaction of $t$ - $\mathrm{Bu}_{2} \mathrm{PH}$ and methacrylonitrile, under pseudo-first-order conditions in methacrylonitrile. The pseudofirst-order rate constant $3.26 \times 10^{-4} \mathrm{~s}^{-1}$ is determined from the slope of the curve from a least-squares fit to the data.

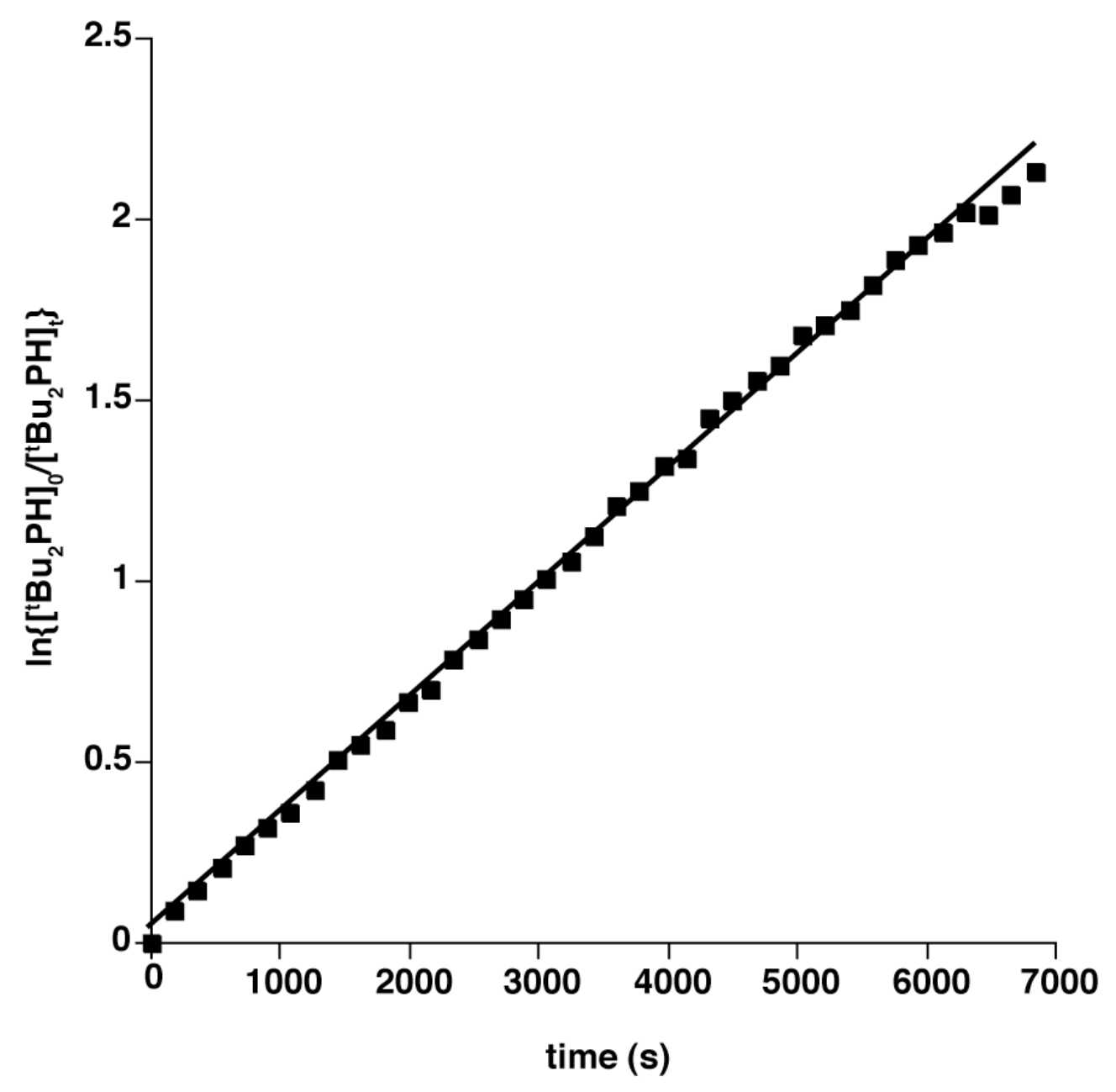


Figure S2. Representative second-order plot $\left(\ln \left\{[\right.\right.$ methacrylonitrile $\left.] /\left[t-\mathrm{Bu}_{2} \mathrm{PH}\right]\right\}$ vs time $)$ of the $\left[\mathrm{Ni}\left(\mathrm{K}^{3}\right.\right.$-Pigiphos $)($ methacrylonitrile $\left.)\right]\left[\mathrm{BAr}_{\mathrm{f}}\right]_{2}$-catalyzed reaction of $t-\mathrm{Bu}_{2} \mathrm{PH}$ and methacrylonitrile, under conditions of excess methacrylonitrile. The second order rate constants is determined from the equation $\mathrm{k}^{\prime}=\operatorname{slope} / \Delta_{0},\left(\Delta_{0}=[\text { methacrylonitrile }]_{0}-[t\right.$ $\left.\mathrm{Bu}_{2} \mathrm{PH}\right]_{0}$ ), and the slope is determined by a least squares fit of a curve to the experimental data.

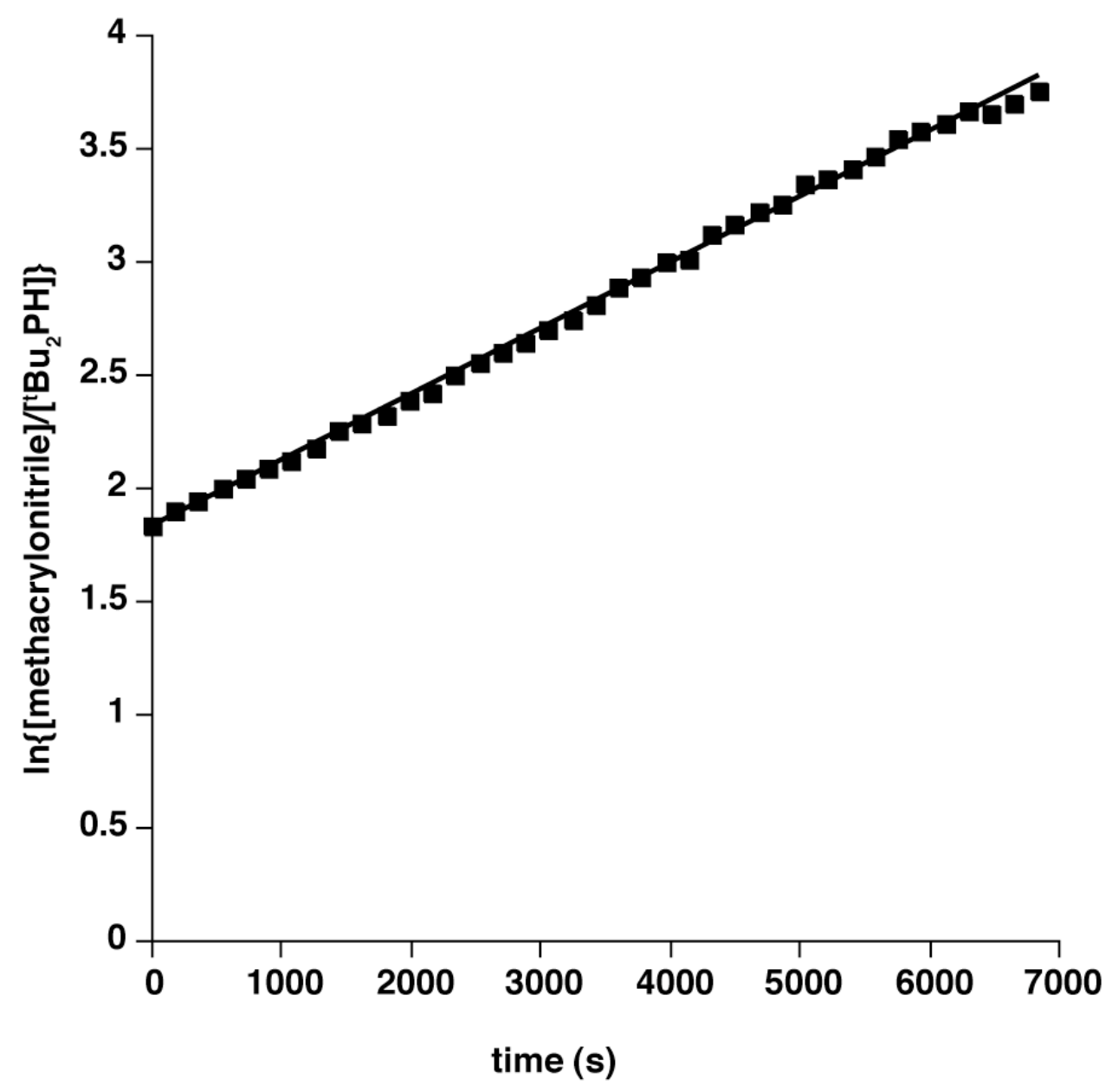


Figure S3. Ortep diagram of $\left[\mathrm{PdCl}\left(\kappa^{2}-(R)-\left(\mathrm{C}_{10} \mathrm{H}_{6}\right) \mathrm{CHMe}\left(\mathrm{NMe}_{2}\right)\right)\left(\kappa^{1}-(S)-t-\right.\right.$ $\left.\left.\mathrm{Bu}_{2} \mathrm{PCH}_{2} \mathrm{CH}\left(\mathrm{CH}_{3}\right) \mathrm{CN}\right)\right],(\boldsymbol{R}, \boldsymbol{S})-\mathbf{S 2}$, showing that the absolute configuration of the stereogenic carbon of $t-\mathrm{Bu}_{2} \mathrm{PCH}_{2} C H M e C N$ is $S$. Hydrogens, with the exception of the hydrogens on the stereogenic carbons atoms, are omitted for clarity (thermal ellipsoids are at the $50 \%$ probability level).

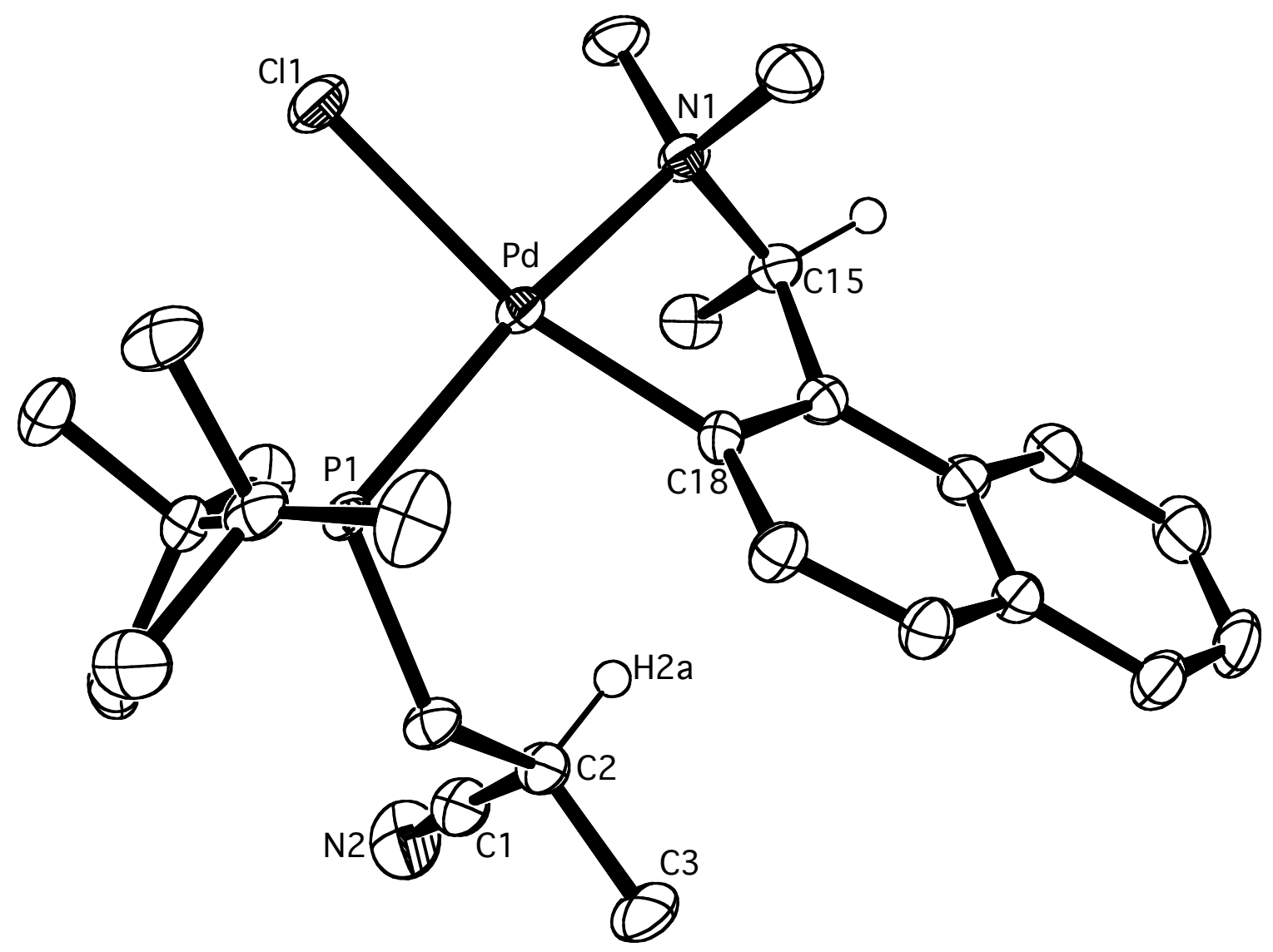


Figure S4. Ortep diagram of $\left[\mathrm{PdCl}\left(\kappa^{2}-(R)-\left(\mathrm{C}_{10} \mathrm{H}_{6}\right) \mathrm{CHMe}\left(\mathrm{NMe}_{2}\right)\right)\left(\kappa^{1}-(S)-(1-\right.\right.$ $\left.\left.\mathrm{Ad})_{2} \mathrm{PCH}_{2} \mathrm{CH}\left(\mathrm{CH}_{3}\right) \mathrm{CN}\right)\right],(\boldsymbol{R}, \boldsymbol{S})$-S4, demonstrating that the absolute configuration of (1$\mathrm{Ad}_{2} \mathrm{PCH}_{2} \mathrm{CHMeCN}$ is $S$. Hydrogens, with the exception of the hydrogens on the stereogenic carbons atoms, are omitted for clarity. Two independent molecules cocrystallize and were independently refined, and both molecules were the enantiomer with the absolute configuration $R, S$. Although the metrical parameters differ slightly, only one independent molecule is shown here (thermal ellipsoids are at the 50\% probability level).

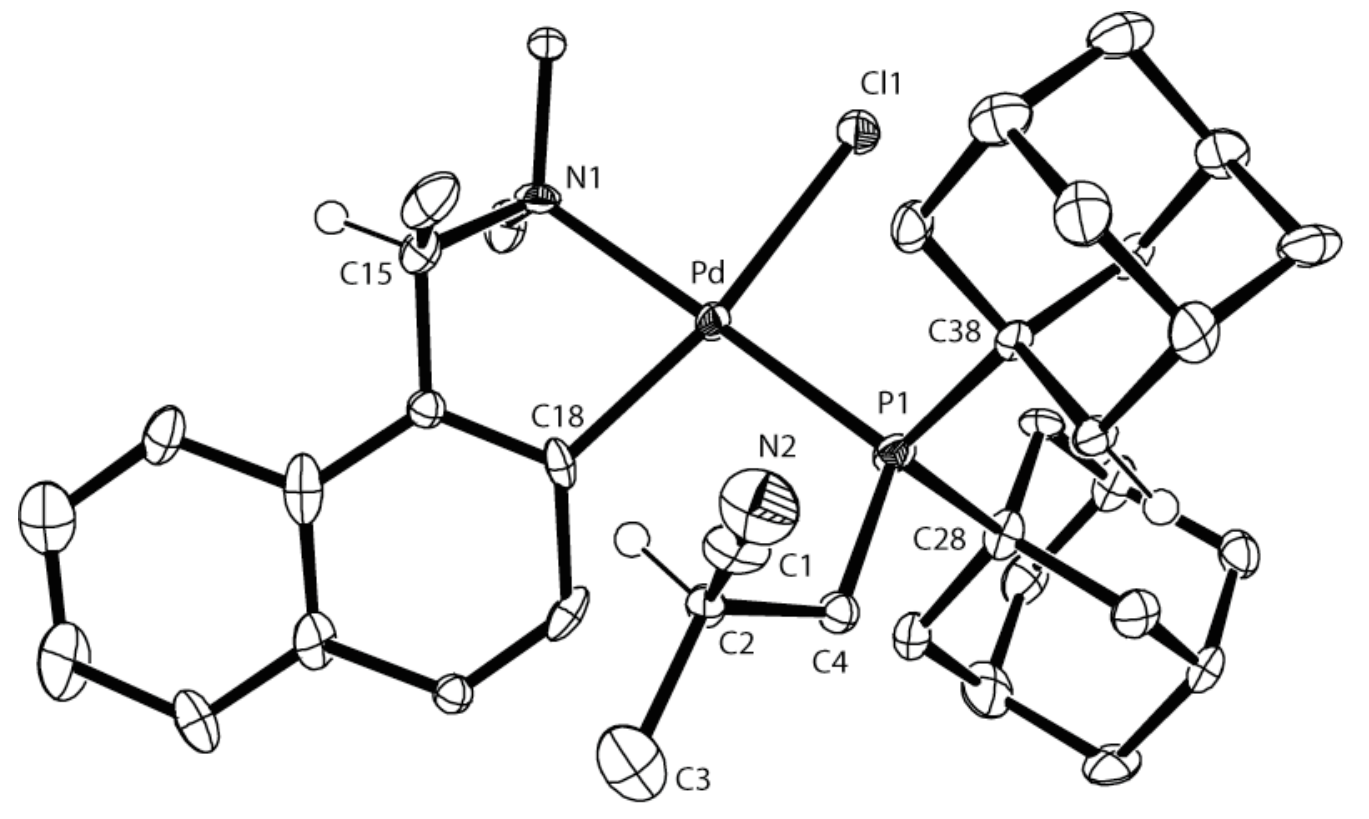

\section{Reference}

1. (corresponds to ref. 23a in the paper): Gaussian 03, Revision C.02, Frisch, M. J.; Trucks, G. W.; Schlegel, H. B.; Scuseria, G. E.; Robb, M. A.; Cheeseman, J. R.; Montgomery, Jr., J. A.; Vreven, T.; Kudin, K. N.; Burant, J. C.; Millam, J. M.; Iyengar, S. S.; Tomasi, J.; Barone, V.; Mennucci, B.; Cossi, M.; Scalmani, G.; Rega, N.; Petersson, G. A.; Nakatsuji, H.; Hada, M.; Ehara, M.; Toyota, K.; Fukuda, R.; Hasegawa, J.; Ishida, M.; Nakajima, T.; Honda, Y.; Kitao, O.; Nakai, H.; Klene, M.; Li, X.; Knox, J. E.; Hratchian, H. P.; Cross, J. B.; Bakken, V.; Adamo, C.; Jaramillo, J.; Gomperts, R.; Stratmann, R. E.; Yazyev, O.; Austin, A. J.; Cammi, R.; Pomelli, C.; Ochterski, J. W.; Ayala, P. Y.; Morokuma, K.; Voth, G. A.; Salvador, P.; Dannenberg, J. J.; Zakrzewski, V. G.; Dapprich, S.; Daniels, A. D.; Strain, M. C.; Farkas, O.; Malick, D. K.; Rabuck, A. D.; Raghavachari, K.; Foresman, J. B.; Ortiz, J. V.; Cui, Q.; Baboul, A. G.; Clifford, S.; Cioslowski, J.; Stefanov, B. B.; Liu, G.; Liashenko, A.; Piskorz, P.; Komaromi, I.; Martin, R. L.; Fox, D. J.; Keith, T.; Al-Laham, M. A.; Peng, C. Y.; Nanayakkara, A.; Challacombe, M.; Gill, P. M. W.; Johnson, B.; Chen, W.; Wong, M. W.; Gonzalez, C.; and Pople, J. A.; Gaussian, Inc., Wallingford CT, 2004. 